\title{
Article
}

\section{The hyper order and fixed points of solutions of a class of linear differential equations}

\author{
Nour el imane Khadidja CHERIET ${ }^{1}$ and Benharrat BELAÏDI ${ }^{2, *}$ \\ 1 Department of Mathematics, Ibnou-Khaldoun University, Tiaret-Algeria. \\ 2 Department of Mathematics, Laboratory of Pure and Applied Mathematics, University of Mostaganem (UMAB), B. \\ P. 227 Mostaganem-Algeria. \\ * Correspondence: benharrat.belaidi@univ-mosta.dz
}

Academic Editor: Alberto Cabada

Received: 11 June 2021; Accepted: 23 July 2021; Published: 12 August 2021.

Abstract: In this paper, we precise the hyper order of solutions for a class of higher order linear differential equations and investigate the exponents of convergence of the fixed points of solutions and their first derivatives for the second order case. These results generalize those of Nan Li and Lianzhong Yang and of Chen and Shon.

Keywords: Linear differential equations; Hyper order; Fixed points.

MSC: 34M10, 30D35.

\section{Introduction}

I

$\mathrm{n}$ this paper, we use standard notations from the value distribution theory of meromorphic functions (see [1-3]). We suppose that $f$ is a meromorphic function in whole complex plane $\mathbb{C}$. In addition, we denote the order of growth of $f$ by $\rho(f)$, and use the notation $\rho_{2}(f)$ to denote the hyper-order of $f$, defined by

$$
\rho_{2}(f)=\limsup _{r \rightarrow+\infty} \frac{\log \log T(r, f)}{\log r}
$$

where $T(r, f)$ is the Nevanlinna characteristic function of $f$.

To give the precise estimate of fixed points, we denote the exponent of convergence of fixed points by $\tau(f)$, which is defined by

$$
\tau(f)=\lambda(f-z)=\limsup _{r \rightarrow+\infty} \frac{\log N\left(r, \frac{1}{f-z}\right)}{\log r}
$$

and the hyper-exponent of convergence of fixed points and distinct fixed points are denoted by $\tau_{2}(f)$ and $\bar{\tau}_{2}(f)$ and are defined by

$$
\tau_{2}(f)=\lambda_{2}(f-z)=\limsup _{r \rightarrow+\infty} \frac{\log \log N\left(r, \frac{1}{f-z}\right)}{\log r},
$$

and

$$
\bar{\tau}_{2}(f)=\bar{\lambda}_{2}(f-z)=\limsup _{r \rightarrow+\infty} \frac{\log \log \bar{N}\left(r, \frac{1}{f-z}\right)}{\log r},
$$

respectively, where $N\left(r, \frac{1}{f-z}\right)$ and $\bar{N}\left(r, \frac{1}{f-z}\right)$ are respectively the integrated counting function of fixed points and distinct fixed points of $f$. We denote the exponent of convergence of zeros (distinct zeros) of $f$ by $\lambda(f)$ $(\bar{\lambda}(f))$ and the hyper-exponent of convergence of zeros (distinct zeros) of $f$ by $\lambda_{2}(f)\left(\bar{\lambda}_{2}(f)\right)$.

Consider the second-order homogeneous linear differential equation

$$
f^{\prime \prime}+P\left(e^{z}\right) f^{\prime}+Q\left(e^{z}\right) f=0,
$$


where $P(w)$ and $Q(w)$ are not constants polynomials in $w=e^{z}(z \in \mathbb{C})$. It's well-known that every solution of Equation (1) is entire.

Suppose $f \not \equiv 0$ is a solution of (1). If $f$ satisfies the condition

$$
\limsup _{r \rightarrow+\infty} \frac{\log T(r, f)}{r}=0
$$

then we say that $f$ is a nontrivial subnormal solution of (1), and if $f$ satisfies the condition [4],

$$
\limsup _{r \rightarrow+\infty} \frac{\log T(r, f)}{r^{n}}=0
$$

then we say that $f$ is a nontrivial $n$-subnormal solution of (1). In [5], Wittich investigated the subnormal solution of (1), and obtained the form of all subnormal solutions in the following theorem:

Theorem 1. [5] If $f \not \equiv 0$ is a subnormal solution of (1), then $f$ must have the form

$$
f(z)=e^{c z}\left(a_{0}+a_{1} e^{z}+\cdots+a_{m} e^{m z}\right),
$$

where $m \geq 0$ is an integer and $c, a_{0}, a_{1}, \ldots, a_{m}$ are constants with $a_{0} a_{m} \neq 0$.

Gundersen and Steinbert [6] refined Theorem 1 and got the following theorem:

Theorem 2. [6] Under the assumption of Theorem 1, the following statements hold:

(i) If $\operatorname{deg} P>\operatorname{deg} Q$ and $Q \not \equiv 0$, then any subnormal solution $f \not \equiv 0$ of (7) must have the form

$$
f(z)=\sum_{k=0}^{m} h_{k} e^{-k z}
$$

where $m \geq 1$ is an integer and $h_{0}, h_{1}, \ldots, h_{m}$ are constants with $h_{0} \neq 0$ and $h_{m} \neq 0$.

(ii) If $\operatorname{deg} P \geq 1$ and $Q \equiv 0$, then any subnormal solution of Equation (7) must be constant.

(iii) If $\operatorname{deg} P<\operatorname{deg} Q$, then the only subnormal solution of (7) is $f \equiv 0$.

Chen and Shon [7] investigated more general equation than (7), and got the following theorem: Set

$$
\begin{gathered}
a_{j}(z)=a_{j d_{j}} z^{d_{j}}+a_{j\left(d_{j}-1\right)} z^{d_{j}-1}+\cdots+a_{j 1} z+a_{j 0},(j=0, \cdots, n), \\
b_{k}(z)=b_{k m_{k}} z^{m_{k}}+b_{k\left(m_{k}-1\right)} z^{m_{k}-1}+\cdots+b_{k 1} z+b_{k 0},(k=0, \cdots, s),
\end{gathered}
$$

where $d_{j} \geq 0(j=0, \cdots, n), m_{k} \geq 0(k=0, \cdots, s)$ are integers, $a_{j d_{j}}, \ldots, a_{j 0} ; b_{k m_{k}}, \ldots, b_{k 0}$ are complex constants such that $a_{j d_{j}} \neq 0, b_{k m_{k}} \neq 0$.

Theorem 3. [7] Let $a_{n}(z), \ldots, a_{1}(z), a_{0}(z), b_{s}(z), \ldots, b_{1}(z), b_{0}(z)$ be polynomials and satisfy (2) and (3), and $a_{n}(z) b_{s}(z) \neq 0$. Suppose that $P^{*}\left(e^{z}\right)=a_{n}(z) e^{n z}+\cdots+a_{1}(z) e^{z}+a_{0}(z), Q^{*}\left(e^{z}\right)=b_{s}(z) e^{s z}+\cdots+b_{1}(z) e^{z}+b_{0}(z)$. If $n<s$, then every solution $f(\not \equiv)$ of equation

$$
f^{\prime \prime}+P^{*}\left(e^{z}\right) f^{\prime}+Q^{*}\left(e^{z}\right) f=0
$$

satisfies $\rho_{2}(f)=1$.

Many authors investigated the growth of solutions and the existence of subnormal solutions for some class of higher order linear differential equations (see [4,7-13]). For the higher-order linear homogeneous differential equation

$$
f^{(k)}+P_{k-1}\left(e^{z}\right) f^{(k-1)}+\cdots+P_{0}\left(e^{z}\right) f=0,
$$


where $P_{j}\left(e^{z}\right)(j=0, \cdots, k-1)$ are polynomials in $z$, Yang and Li [11] generalized the result of Theorem 2 to the higher order and obtained the following results: Set

$$
a_{j m_{j}}(z)=a_{j m_{j} d_{j m j}} z^{d_{j m_{j}}}+a_{j m_{j}\left(d_{j m_{j}}-1\right)} z^{d_{j m_{j}}-1}+\cdots+a_{j m_{j} 1} z+a_{j m_{j} 0}
$$

where $d_{j m_{j}} \geq 0(j=0, \cdots, k-1)$ are integers, $a_{j m_{j} d_{j m j}}, \ldots, a_{j m_{j} 0}$ are complex constants, $a_{j m_{j} d_{j m j}} \neq 0$.

Theorem 4. [11] Let $a_{j m_{j}}(z)$ be polynomials and satisfy (5). Suppose that

$$
P_{j}\left(e^{z}\right)=a_{j m_{j}}(z) e^{m_{j} z}+\cdots+a_{j 1}(z) e^{z},
$$

where $a_{j m_{j}}(z) \not \equiv 0$. If there exists an integer $s(s \in\{0, \cdots, k-1\})$ satisfying

$$
m_{s}>\max \left\{m_{j}: j=0, \cdots, s-1, s+1, \cdots, k-1\right\}=m,
$$

then every solution $f \not \equiv 0$ of Equation (4) satisfies $\rho_{2}(f)=1$ if one of the following condition holds:

(i) $s=0$ or 1 .

(ii) $s \geq 2$ and $\operatorname{deg} a_{0 j}(z)>\operatorname{deg} a_{i j}(z)(i \neq 0)$.

Theorem 5. [11] Under the assumption of Theorem 4, if $z P_{0}\left(e^{z}\right)+P_{1}\left(e^{z}\right) \not \equiv 0$, then we have every solution $f \not \equiv 0$ of Equation (4) satisfies

$$
\tau_{2}(f)=\bar{\tau}_{2}(f)=\rho_{2}(f)=1 .
$$

In particular, they also investigated the exponents of convergence of the fixed points of solutions and their first derivatives for a second order Equation (1) and obtained the following theorem:

Theorem 6. [11] Let $a_{n}(z), \ldots, a_{1}(z), b_{s}(z), \ldots, b_{1}(z)$ be polynomials and satisfy (2) and (3), and $a_{n}(z) b_{s}(z) \neq 0$. Suppose that $P\left(e^{z}\right)=a_{n}(z) e^{n z}+\cdots+a_{1}(z) e^{z}, Q\left(e^{z}\right)=b_{s}(z) e^{s z}+\cdots+b_{1}(z) e^{z}$. If $n \neq s$, then every solution $f$ $(\not \equiv 0)$ of Equation (1) satisfy $\lambda(f-z)=\lambda\left(f^{\prime}-z\right)=\rho(f)=\infty$ and $\lambda_{2}(f-z)=\lambda_{2}\left(f^{\prime}-z\right)=\rho_{2}(f)=1$.

Thus, it is natural to ask what will happen if we change $\exp \{z\}$ in the coefficients of (4) into exp $\{A(z)\}$ ? In this paper, we consider the above problem to Theorems $3,4,5$ and 6, we obtain the following results: We set

$$
A(z)=c_{n} z^{n}+c_{n-1} z^{n-1}+\cdots+c_{1} z+c_{0}
$$

where $n \geq 1$ is an integer and $c_{0}, \ldots, c_{n}$ are complex constants such that $\operatorname{Rec}_{n}>0$, throughout the rest of this paper.

Theorem 7. Let $a_{j m_{j}}(z)$ be polynomials and satisfy (5). Suppose that

$$
P_{j}\left(e^{A(z)}\right)=a_{j m_{j}}(z) e^{m_{j} A(z)}+\cdots+a_{j 1}(z) e^{A(z)},
$$

where $a_{j m_{j}}(z) \not \equiv 0$. If there exists an integer $s(s \in\{0, \cdots, k-1\})$ satisfying

$$
m_{s}>\max \left\{m_{j}: j=0, \cdots, s-1, s+1, \cdots, k-1\right\}=m,
$$

then every solution $f \not \equiv 0$ of equation

$$
f^{(k)}+P_{k-1}\left(e^{A(z)}\right) f^{(k-1)}+\cdots+P_{0}\left(e^{A(z)}\right) f=0
$$

satisfies $\rho(f)=\infty$ and $\rho_{2}(f)=n$ if one of the following condition holds:

(i) $s=0$ or 1 .

(ii) $s \geq 2$ and $\operatorname{deg} a_{0 j}(z)>\operatorname{deg} a_{i j}(z)(i \neq 0)$. 
Example 1. Let $f=e^{z^{z^{2}}}$ be a solution of the equation

$$
f^{(4)}-2 z e^{z^{2}} f^{(3)}-12 z^{2} e^{z^{2}} f^{\prime \prime}-24 z^{3} e^{z^{2}} f^{\prime}-\left[24 z^{2} e^{3 z^{2}}+\left(96 z^{2}+12\right) e^{2 z^{2}}+\left(16 z^{4}+48 z^{2}+12\right) e^{z^{2}}\right] f=0 .
$$

Set

$$
\begin{aligned}
& P_{3}\left(e^{A(z)}\right)=a_{3,1}(z) e^{A(z)}=-2 z e^{z^{2}}, \\
& P_{2}\left(e^{A(z)}\right)=a_{2,1}(z) e^{A(z)}=-12 z^{2} e^{z^{2}}, \\
& P_{1}\left(e^{A(z)}\right)=a_{1,1}(z) e^{A(z)}=-24 z^{3} e^{z^{2}}, \\
& P_{0}\left(e^{A(z)}\right)=a_{0,3}(z) e^{3 A(z)}+a_{0,2}(z) e^{2 A(z)}+a_{0,1}(z) e^{A(z)}=-24 z^{2} e^{3 z^{2}}-\left(96 z^{2}+12\right) e^{2 z^{2}}-\left(16 z^{4}+48 z^{2}+12\right) e^{z^{2}} .
\end{aligned}
$$

We remark that $s=0$ and $m_{0}=3>\max \left\{m_{j}: j=1,2,3\right\}=m=1$. Obviously, the conditions of Theorem 7 are satisfied, we see that $\rho(f)=\infty$ and $\rho_{2}(f)=n=2$.

Remark 1. Very recently, Li et al., [4] have investigated $n$ subnormal solutions of the Equation (7) with

$$
P_{j}\left(e^{A(z)}\right)=a_{j m_{j}} e^{m_{j} A(z)}+\cdots+a_{j 1} e^{A(z)}(j=0, \ldots, k-1),
$$

where $a_{j m_{j}}, \cdots, a_{j 1}(j=0, \ldots, k-1)$ are complex constants instead of polynomials and obtained some results concerning their growth.

Corollary 1. Under the assumption of Theorem 7 , if $z P_{0}\left(e^{A(z)}\right)+P_{1}\left(e^{A(z)}\right) \not \equiv 0$, then we have every solution $f \not \equiv 0$ of Equation (4) satisfies

$$
\tau(f)=\bar{\tau}(f)=\rho(f)=\infty \text { and } \tau_{2}(f)=\bar{\tau}_{2}(f)=\rho_{2}(f)=n .
$$

In particular, we also investigate the exponents of convergence of the fixed points of solutions and their first derivatives for a second order equation

$$
f^{\prime \prime}+P\left(e^{A(z)}\right) f^{\prime}+Q\left(e^{A(z)}\right) f=0,
$$

and we obtain the following theorems:

Theorem 8. Let $a_{p}(z), \ldots, a_{1}(z), b_{s}(z), \ldots, b_{1}(z)$ be polynomials and satisfy (2) and (3), and $a_{p}(z) b_{s}(z) \neq 0$. Suppose that $P\left(e^{A(z)}\right)=a_{p}(z) e^{p A(z)}+\cdots+a_{1}(z) e^{A(z)}, Q\left(e^{A(z)}\right)=b_{s}(z) e^{s A(z)}+\cdots+b_{1}(z) e^{A(z)}$. If $p \neq s$, then every solution $f(\not \equiv 0)$ of Equation (8) satisfies $\lambda(f-z)=\lambda\left(f^{\prime}-z\right)=\rho(f)=\infty$ and $\lambda_{2}(f-z)=\lambda_{2}\left(f^{\prime}-z\right)=\rho_{2}(f)=$ $n$.

Example 2. Let $f=e^{z^{z^{2}}}$ be a solution of the equation

$$
f^{\prime \prime}-3 z e^{z^{2}} f^{\prime}+\left[2 z^{2} e^{2 z^{2}}-\left(4 z^{2}+2\right) e^{z^{2}}\right] f=0 .
$$

Set

$$
\begin{aligned}
& P\left(e^{A(z)}\right)=a_{1}(z) e^{A(z)}=-3 z e^{z^{2}} \\
& Q\left(e^{A(z)}\right)=b_{2}(z) e^{2 A(z)}+b_{1}(z) e^{A(z)}=2 z^{2} e^{2 z^{2}}-\left(4 z^{2}+2\right) e^{z^{2}} .
\end{aligned}
$$

It is clear that the conditions of Theorem 8 are satisfied with $p=1 \neq s=2$, we see that $\lambda\left(e^{e^{z^{2}}}-z\right)=$ $\lambda\left(2 z e^{z^{2}} e^{e^{z^{2}}}-z\right)=\rho(f)=\infty$ and $\lambda_{2}\left(e^{e^{z^{2}}}-z\right)=\lambda_{2}\left(2 z e^{z^{2}} e^{e^{z^{2}}}-z\right)=\rho_{2}(f)=n=2$. 
Remark 2. If $p=s$, then the conclusions of Theorem 8 does not hold. For instance, consider the following equation

$$
\begin{aligned}
f^{\prime \prime}+ & \left(\left(z^{4}+2 i z\right) e^{2(1+5 i) z^{3}+2 z}+\left(-z^{2}+(2-i) z\right) e^{(1+5 i) z^{3}+z}\right) f^{\prime} \\
& -\left(\left(z^{3}+2 i\right) e^{2(1+5 i) z^{3}+2 z}+(-z+2-i) e^{(1+5 i) z^{3}+z}\right) f=0
\end{aligned}
$$

We can easily see that (9) has solution $f(z)=z$ which satisfies $\rho(f)=0 \neq \infty$ and $\rho_{2}(f)=0 \neq n=3$. In this example, we have $p=s=2, A(z)=(1+5 i) z^{3}+z, a_{2}(z)=z^{4}+2 i z, a_{1}(z)=-z^{2}+(2-i) z$, $b_{2}(z)=-\left(z^{3}+2 i\right)$ and $b_{1}(z)=-(-z+2-i)$.

Theorem 9. Let $a_{p}(z), \ldots, a_{1}(z), a_{0}(z), b_{s}(z), \ldots, b_{1}(z), b_{0}(z)$ be polynomials and satisfy (2) and (3), and $a_{p}(z) b_{s}(z) \neq$ 0. Suppose that

$$
\begin{aligned}
& P^{*}\left(e^{A(z)}\right)=a_{p}(z) e^{p A(z)}+\cdots+a_{1}(z) e^{A(z)}+a_{0}(z), \\
& Q^{*}\left(e^{A(z)}\right)=b_{s}(z) e^{s A(z)}+\cdots+b_{1}(z) e^{A(z)}+b_{0}(z) .
\end{aligned}
$$

If $p<s$, then every solution $f(\not \equiv 0)$ of equation

$$
f^{\prime \prime}+P^{*}\left(e^{A(z)}\right) f^{\prime}+Q^{*}\left(e^{A(z)}\right) f=0
$$

satisfies $\rho(f)=\infty$ and $\rho_{2}(f)=n$.

Example 3. Let $f=e^{z} e^{e^{z}}$ be a solution of the equation

$$
f^{\prime \prime}+\left(e^{z+1}-3\right) f^{\prime}+\left[\left(-e^{-2}-e^{-1}\right) e^{2(z+1)}-e^{z+1}+2\right] f=0 .
$$

Set

$$
\begin{aligned}
& P^{*}\left(e^{A(z)}\right)=a_{1}(z) e^{A(z)}+a_{0}(z)=e^{z+1}-3 \\
& Q^{*}\left(e^{A(z)}\right)=b_{2}(z) e^{2 A(z)}+b_{1}(z) e^{A(z)}+b_{0}(z)=\left(-e^{-2}-e^{-1}\right) e^{2(z+1)}-e^{z+1}+2
\end{aligned}
$$

It is clear that the conditions of Theorem 9 are satisfied with $p=1<s=2$, here we have $\rho(f)=\infty$ and $\rho_{2}(f)=n=1$.

Remark 3. If $p \geq s$, then the conclusions of Theorem 9 does not hold. For instance, consider the following equation

$$
\begin{aligned}
f^{\prime \prime}- & \left(\left(2 z^{2}+3 z\right) e^{(1-i) z^{2}+2 z+i}+i z^{3}-z^{2}+(1+i) z\right) f^{\prime} \\
+ & \left((2 z+3) e^{(1-i) z^{2}+2 z+i}+i z^{2}-z+1+i\right) f=0 .
\end{aligned}
$$

It is easy to see that (11) has solution $f(z)=z$ which satisfies $\rho(f)=0 \neq \infty$ and $\rho_{2}(f)=0 \neq n=2$. In this example, we have $p=s=1, A(z)=(1-i) z^{2}+2 z+i, a_{1}(z)=-\left(2 z^{2}+3 z\right), a_{0}(z)=-\left(i z^{3}-z^{2}+(1+i) z\right)$, $b_{1}(z)=2 z+3$ and $b_{0}(z)=i z^{2}-z+1+i$.

Remark 4. Setting $c_{n}=1, c_{n-1}=\cdots=c_{0}=0$ and $n=1$, in Theorem 7, Corollary 1, Theorem 8 and Theorem 9, we obtain Theorem 4, Theorem 5, Theorem 6 and Theorem 3 respectively.

\section{Auxiliary Lemmas}

Recall that

$$
A(z)=c_{n} z^{n}+c_{n-1} z^{n-1}+\cdots+c_{0,}, c_{l}=\alpha_{l} e^{i \theta_{l}}, z=r e^{i \theta}, \operatorname{Rec}_{n}>0,
$$


we set $\delta_{l}(A, \theta)=\operatorname{Re}\left(c_{l}\left(e^{i \theta}\right)^{l}\right)=\alpha_{l} \cos \left(\theta_{l}+l \theta\right)$, and $H_{l, 0}=\left\{\theta \in[0,2 \pi): \delta_{l}(A, \theta)=0\right\}, H_{l,+}=\{\theta \in[0,2 \pi)$ : $\left.\delta_{l}(A, \theta)>0\right\}, H_{l,-}=\left\{\theta \in[0,2 \pi): \delta_{l}(A, \theta)<0\right\}$, for $l=1, \cdots, n$, throughout the rest of this paper. Obviously, if $\delta_{n}(A, \theta) \neq 0$, as $r \rightarrow+\infty$, we get

$$
\left|e^{A(z)}\right|=e^{\delta_{n}(A, \theta) r^{n}+\cdots+\delta_{1}(A, \theta) r+\operatorname{Rec} 0}=e^{\delta_{n}(A, \theta) r^{n}(1+o(1))} .
$$

Lemma 1. [3] Let $f_{j}(z)(j=1, \cdots, n)(n \geq 2)$ be meromorphic functions, $g_{j}(z)(j=1, \cdots, n)$ be entire functions, and satisfy

(i) $\sum_{j=1}^{n} e^{g_{j}(z)} \equiv 0$;

(ii) when $1 \leq j \leq k \leq n$, then $g_{i}(z)-g_{k}(z)$ is not a constant;

(iii) when $1 \leq j \leq n, 1 \leq h \leq k \leq n$,

then

$$
T\left(r, f_{j}\right)=o\left\{T\left(r, e^{g_{h}-g_{k}}\right)\right\} \quad(r \rightarrow+\infty, r \notin E),
$$

where $E \subset(1, \infty)$ is of finite linear measure or logarithmic measure. Also, $f_{j}(z) \equiv 0(j=1, \cdots, n)$.

Lemma 2. Let $A(z), P_{j}\left(e^{A(z)}\right), m_{j}, m_{s}, m$ and $a_{i j}(z)$ satisfy the hypotheses of Theorem 7 , then Equation (7) has no constant polynomial solution.

Proof. Suppose that $f_{0}(z)=b_{l} z^{l}+\cdots+b_{1} z+b_{0}(l \geq 1)$ is a nonconstant polynomial solution of (7), where $b_{l} \neq 0, \cdots, b_{0}$ are complex constants.

If $l \geq s$, then $f^{(s)} \not \equiv 0$. Taking $z=r$, we have

$$
\left|e^{A(z)}\right|=\left|e^{A(r)}\right|=\left|e^{c_{n} r^{n}+c_{n-1} r^{n-1}+\cdots+c_{0}}\right|=e^{\operatorname{Rec}_{n} r^{n}+\operatorname{Rec}_{n-1} r^{n-1}+\cdots+\operatorname{Rec}_{0}}=e^{\operatorname{Rec}_{n} r^{n}(1+o(1))} .
$$

Substituting $f_{0}$ into (7) and using (13), we conclude that

$$
\begin{aligned}
& \left|a_{s m_{s} d s m_{s}} r^{d_{s m_{s}}} e^{m_{s} \operatorname{Rec}_{n} r^{n}(1+o(1))}\right| b_{l} l(l-1) \cdots(l-s+1)\left|r^{l-s}(1+o(1)) \leq\right|-P_{s}\left(e^{A(r)}\right) f_{0}^{(s)}(r) \mid \\
& \leq\left|f^{(k)}(r)\right|+\left|P_{k-1}\left(e^{A(r)}\right) f_{0}^{(k-1)}(r)\right|+\cdots+\left|P_{s+1}\left(e^{A(r)}\right) f_{0}^{(s+1)}(r)\right|+\left|P_{s-1}\left(e^{A(r)}\right) f_{0}^{(s-1)}(r)\right|+\cdots+\left|P_{0}\left(e^{A(r)}\right) f_{0}(r)\right| \\
& \leq M_{0} r^{d} e^{m \operatorname{Rec}_{n} r^{n}(1+o(1))}(1+o(1))
\end{aligned}
$$

where $d=\max \left\{d_{j m_{j}}: j=0, \cdots, s-1, s+1, \cdots, k-1\right\}$ and $M_{0}>0$ is some constant. Since $m_{s}>m$, we see that (3) is a contradiction. Obviously, when $s=0$ or 1, we can get that the Equation (7) has nonconstant polynomial solution from the above process. If $l<s$, then

$$
P_{l}\left(e^{A(z)}\right) f_{0}^{(l)}(z)+\cdots+P_{0}\left(e^{A(z)}\right) f_{0}(z)=0 .
$$

Set $\max \left\{m_{j}: j=0, \cdots, l\right\}=h$. If $m_{j}<h$, then we can rewrite

$$
P_{j}\left(e^{A(z)}\right)=a_{j h}(z) e^{h A(z)}+\cdots+a_{j\left(m_{j}+1\right)}(z) e^{\left(m_{j}+1\right) A(z)}+a_{j m_{j}}(z) e^{m_{j} A(z)}+\cdots+a_{j 1}(z) e^{A(z)}
$$

for $j=0, \cdots, l$, where $a_{j h}(z)=\cdots=a_{j\left(m_{j}+1\right)}(z)=0$. Thus, we conclude by (15) that

$$
\begin{aligned}
& {\left[a_{l h}(z) f_{0}^{(l)}+a_{(l-1) h}(z) f_{0}^{(l-1)}+\cdots+a_{0 h}(z) f_{0}\right] e^{h A(z)}+\cdots+\left[a_{l j}(z) f_{0}^{(l)}+a_{(l-1) j}(z) f_{0}^{(l-1)}\right.} \\
& \left.+\cdots+a_{0 j}(z) f_{0}\right] e^{j A(z)}+\cdots+\left[a_{l 1}(z) f_{0}^{(l)}+a_{(l-1) 1}(z) f_{0}^{(l-1)}+\cdots+a_{01}(z) f_{0}\right] e^{A(z)}=0 .
\end{aligned}
$$

Set

$$
Q_{j}(z)=a_{l j}(z) f_{0}^{(l)}+a_{(l-1) j}(z) f_{0}^{(l-1)}+\cdots+a_{0 j} f_{0} \quad(j=1, \cdots, h) .
$$

Since $f_{0}$ and $a_{i j}(z)$ are polynomials, we see that

$$
m\left(r, Q_{j}\right)=o\left\{m\left(r, e^{(\alpha-\beta) A(z))}\right\}, \quad(1 \leq \beta<\alpha \leq h) .\right.
$$


By Lemma 1 and (16) -(18), we conclude that

$$
Q_{1}(z) \equiv Q_{2}(z) \equiv \cdots \equiv Q_{h}(z) \equiv 0 .
$$

Since $\operatorname{deg} f_{0}>\operatorname{deg} f_{0}^{\prime}>\cdots>\operatorname{deg} f_{0}^{(l)}$ and $\operatorname{deg} a_{0 j}(z)>\operatorname{deg} a_{i j}(z)(i \neq 0)$, so by (16) and (19), we get a contradiction.

Lemma 3. $[14,15]$ Let $f(z)$ be an entire function and suppose that $\left|f^{(k)}(z)\right|$ is unbounded on some ray $\arg z=\theta$. Then, there exists an infinite sequence of points $z_{m}=r_{m} e^{i \theta}(m=1,2, \cdots)$, where $r_{m} \rightarrow+\infty$ such that $f^{(k)}\left(z_{m}\right) \rightarrow \infty$ and

$$
\left|\frac{f^{(j)}\left(z_{m}\right)}{f^{(k)}\left(z_{m}\right)}\right| \leq\left|z_{m}\right|^{k-j}(1+o(1)) \quad(j=0, \cdots, k-1) .
$$

Lemma 4. [16] Let $f(z)$ be a transcendental meromorphic function of finite order $\rho$. Let $\Gamma=$ $\left\{\left(k_{1}, j_{1}\right),\left(k_{2}, j_{2}\right), \cdots,\left(k_{m}, j_{m}\right)\right\}$ denote a set of distinct pairs of integers satisfying $k_{i}>j_{i} \geq 0(i=1,2, \cdots, m)$ and let $\varepsilon>0$ be a given constant. Then, there exists a set $E_{1} \subset[0,2 \pi)$ that has linear measure zero such that if $\theta \in[0,2 \pi) \backslash E_{1}$, then there is a constant $R_{1}=R_{1}(\theta)>1$ such that for all $z$ satisfying $\arg z=\theta$ and $|z| \geq R_{1}$ and for all $(k, j) \in \Gamma$, we have

$$
\left|\frac{f^{(k)}(z)}{f^{(j)}(z)}\right| \leq|z|^{(k-j)(\rho-1+\varepsilon)} .
$$

Lemma 5. [17] Let $f(z)$ be an entire function with $\rho(f)=\rho<\infty$. Suppose that there exists a set $E_{2} \subset[0,2 \pi)$ that has linear measure zero, such that for any ray $\arg z=\theta_{0} \in[0,2 \pi) \backslash E_{2},\left|f\left(r e^{i \theta_{0}}\right)\right| \leq M r^{k}\left(M=M\left(\theta_{0}\right)>0\right.$ is a constant and $k>0$ is a constant independent of $\left.\theta_{0}\right)$. Then $f(z)$ is a polynomial with $\operatorname{deg} f \leq k$.

Lemma 6. [16] Let $f$ be a transcendental meromorphic function, and $\alpha>1$ be a given constant. Then, there exists a set $E_{3} \subset(1, \infty)$ with finite logarithmic measure and a constant $C>0$ that depends only on $\alpha$ and $i, j(i, j \in \mathbb{N})$, such that for all $z$ satisfying $|z|=r \notin E_{3} \cup[0,1]$,

$$
\left|\frac{f^{(j)}(z)}{f^{(i)}(z)}\right| \leq C\left(\frac{T(\alpha r, f)}{r}\left(\log ^{\alpha} r\right) \log T(\alpha r, f)\right)^{j-i} .
$$

Remark 5. From the proof of Lemma 6 ([16, Theorem 3]), we can see that the exceptional set $E_{4}$ equals $\{|z|$ : $\left.z \in\left(\cup_{n=1}^{+\infty} O\left(a_{n}\right)\right)\right\}$, where $a_{n}(n=1,2, \cdots)$ denote all zeros and poles of $f^{(i)}$, and $O\left(a_{n}\right)$ denote sufficiently small neighborhoods of $a_{n}$. Hence, if $f(z)$ is a transcendental entire function and $z$ is a point that satisfies $|f(z)|$ to be sufficiently large, then the point $z \notin E_{4}$ satisfies (20). For details, see , [9, Remark 2.10].

Lemma 7. $[10,18]$ Let $A_{0}, \cdots, A_{k-1}$ be entire functions of finite order. If $f(z)$ is a solution of equation

$$
f^{(k)}+A_{k-1} f^{(k-1)}+\cdots+A_{0} f=0,
$$

then $\rho_{2}(f) \leq \max \left\{\rho\left(A_{j}\right): j=0, \cdots, k-1\right\}$.

Lemma 8. [19] Let $g(z)$ be an entire function of infinite order with the hyper-order $\rho_{2}(g)=\rho$, and let $v(r)$ be the central index of $g$. Then,

$$
\limsup _{r \rightarrow+\infty} \frac{\log \log v(r)}{\log r}=\rho_{2}(g)=\rho .
$$

Lemma 9. [7] Let $f(z)$ be an entire function that satisfies $\rho(f)=\rho(n<\rho<\infty)$; or $\rho(f)=\infty$ and $\rho_{2}=0$; or $\rho_{2}=\alpha(0<\alpha<\infty)$, and a set $E_{5} \subset[1, \infty)$ has a finite logarithmic measure. Then, there exists a sequence $\left\{z_{k}=r_{k} e^{i \theta_{k}}\right\}$ such that $\left|f\left(z_{k}\right)\right|=M\left(r_{k}, f\right), \theta_{k} \in[0,2 \pi), \lim _{k \rightarrow \infty} \theta_{k}=\theta_{0} \in[0,2 \pi), r_{k} \notin E_{5}$, and $r_{k} \rightarrow \infty$, such that

(i) if $\rho(f)=\rho(n<\rho<\infty)$, then for any given $\varepsilon_{1}\left(0<\varepsilon_{1}<\frac{\rho-n}{2}\right)$,

$$
r_{k}^{\rho-\varepsilon_{1}}<v\left(r_{k}\right)<r_{k}^{\rho+\varepsilon_{1}}
$$


(ii) if $\rho(f)=\infty$ and $\rho_{2}(f)=0$, then for any given $\varepsilon_{2}\left(0<\varepsilon_{2}<\frac{1}{2}\right)$, and for any large $M(>0)$, we have, as $r_{k}$ is sufficiently large,

$$
r_{k}^{M}<v\left(r_{k}\right)<\exp \left\{r_{k}^{\varepsilon_{2}}\right\}
$$

(iii) if $\rho_{2}(f)=\alpha(0<\alpha<\infty)$, then for any given $\varepsilon_{3}\left(0<\varepsilon_{3}<\alpha\right)$,

$$
\exp \left\{r_{k}^{\alpha-\varepsilon_{3}}\right\}<v\left(r_{k}\right)<\exp \left\{r_{k}^{\alpha+\varepsilon_{3}}\right\}
$$

Lemma 10. [20] Let $g$ be a non-constant entire function, and let $0<\delta<1$. There exists a set $E_{6} \subset[1, \infty)$ of finite logarithmic measure with the following property. For $r \in[1, \infty) \backslash E_{6}$, the central index $v(r)$ of $g$ satisfies

$$
v(r) \leq(\log M(r, g))^{1+\delta}
$$

Lemma 11. [21,22] Let $A_{0}, \ldots, A_{k-1}, F \not \equiv 0$ be finite order meromorphic functions. If $f$ is a meromorphic solution of the equation

$$
f^{(k)}+A_{k-1} f^{(k-1)}+\cdots+A_{0} f=F,
$$

with $\rho(f)=+\infty$ and $\rho_{2}(f)=\rho$, then $f$ satisfies $\bar{\lambda}(f)=\lambda(f)=\rho(f)=\infty$ and $\bar{\lambda}_{2}(f)=\lambda_{2}(f)=\rho_{2}(f)=\rho$.

Lemma 12. [14] Let $\varphi:[0,+\infty) \rightarrow \mathbb{R}$ and $\psi:[0,+\infty) \rightarrow \mathbb{R}$ be monotone non-decreasing functions such that $\varphi(r) \leq \psi(r)$ for all $r \notin E_{7} \cup[0,1]$, where $E_{7} \subset(1,+\infty)$ is a set of finite logarithmic measure. Let $\gamma>1$ be a given constant. Then there exists a $r_{1}=r_{1}(\gamma)>0$ such that $\varphi(r) \leq \psi(\gamma r)$ for all $r>r_{1}$.

\section{Proofs of the results}

Proof of Theorem 7. Suppose that $f \not \equiv 0$ is a solution of (7), then $f$ is an entire function. By Lemma 2, we see that $f$ is transcendental.

First step. We prove that $\rho(f)=\infty$.

Suppose, to the contrary, that $\rho(f)=\rho<\infty$. By Lemma 4, for any given $\varepsilon>0$, there exists a set $E_{1} \subset[0,2 \pi)$ with linear measure zero, such that if $\theta \in[0,2 \pi) \backslash E_{1}$, then there exists a constant $R_{1}=R_{1}(\theta)>1$, such that for all $z$ satisfying $\arg z=\theta$ and $|z|=r>R_{1}$, we have

$$
\left|\frac{f^{(j)}(z)}{f^{(s)}(z)}\right| \leq r^{(\rho-1+\varepsilon)(j-s)} \quad j=s+1, \cdots, k .
$$

Case 1. Take a ray $\arg z=\theta \in H_{n,+} \backslash E_{1}$, then $\delta_{n}(A, \theta)>0$. We assume that $\left|f^{(s)}\left(r e^{i \theta}\right)\right|$ is bounded on the ray $\arg z=\theta$. If $\left|f^{(s)}\left(r e^{i \theta}\right)\right|$ is unbounded on the ray $\arg z=\theta$, then by Lemma 3 , there exists a sequence $\left\{z_{t}=r_{t} e^{i \theta}\right\}$ such that as $r_{t} \rightarrow+\infty, f^{(s)}\left(z_{t}\right) \rightarrow \infty$ and

$$
\left|\frac{f^{(i)}\left(z_{t}\right)}{f^{(s)}\left(z_{t}\right)}\right| \leq r_{t}^{s-i}(1+o(1)) \leq 2 r_{t}^{s}, \quad i=0, \cdots, s-1
$$

By (7), we get

$$
\left|P_{S}\left(e^{A\left(z_{t}\right)}\right)\right| \leq\left|\frac{f^{(k)}\left(z_{t}\right)}{f^{(s)}\left(z_{t}\right)}\right|+\sum_{j=0 j \neq s}^{k-1}\left|P_{j}\left(e^{A\left(z_{t}\right)}\right)\right| \frac{f^{(j)}(z)}{f^{(s)}(z)} \mid .
$$

For $r_{t} \rightarrow+\infty$, we have

$$
\begin{aligned}
& \left|P_{\mathcal{S}}\left(e^{A\left(z_{t}\right)}\right)\right|=\left|a_{s m_{s}}\left(z_{t}\right) e^{m_{S} A\left(z_{t}\right)}+\cdots+a_{s 1}\left(z_{t}\right) e^{A\left(z_{t}\right)}\right| \\
& \geq\left|a_{s m_{s}}\left(z_{t}\right) e^{m_{s} A\left(z_{t}\right)}\right|-\left|a_{s\left(m_{s}-1\right)}\left(z_{t}\right) e^{m_{s-1} A\left(z_{t}\right)}+\cdots+a_{s 1}\left(z_{t}\right) e^{A\left(z_{t}\right)}\right| \\
& \geq\left|a_{s m_{s}}\left(z_{t}\right) e^{m_{s} A\left(z_{t}\right)}\right|-\left[\left|a_{s\left(m_{s}-1\right)}\left(z_{t}\right) e^{m_{s-1} A\left(z_{t}\right)}\right|+\cdots+\left|a_{s 1}\left(z_{t}\right) e^{A\left(z_{t}\right)}\right|\right] \\
& =\left|a_{s m_{s}} d_{s m_{s}}\right| r_{t}^{d_{s m_{s}}} e^{m_{s} \delta_{n}(A, \theta) r_{t}^{n}(1+o(1))}(1+o(1))-\left[\left|a_{s\left(m_{s}-1\right) d_{s\left(m_{s}-1\right)}}\right| r_{t}^{d_{s\left(m_{s}-1\right)}} e^{\left(m_{s}-1\right) \delta_{n}(A, \theta) r_{t}^{n}(1+o(1))}(1+o(1))\right. \\
& \left.+\cdots+\left|a_{s 1 d_{s 1}}\right| r_{t}^{d_{s 1}} e^{\delta_{n}(A, \theta) r_{t}^{n}(1+o(1))}(1+o(1))\right]
\end{aligned}
$$




$$
\geq \frac{1}{2}\left|a_{s m_{s}} d_{s m_{s}}\right| r_{t}^{d_{s m_{s}}} e^{m_{s} \delta_{n}(A, \theta) r_{t}^{n}(1+o(1))}(1+o(1))
$$

and

$$
\begin{aligned}
\left|P_{j}\left(e^{A\left(z_{t}\right)}\right)\right| & =\left|a_{j m_{j}}\left(z_{t}\right) e^{m_{j} A\left(z_{t}\right)}+\cdots+a_{j 1}\left(z_{t}\right) e^{A\left(z_{t}\right)}\right| \\
& \leq\left|a_{j m_{j} d_{j m_{j}}}\right| r_{t}^{d_{j m_{j}}} e^{m_{j} \delta_{n}(A, \theta) r_{t}^{n}(1+o(1))}(1+o(1))+\cdots+\left|a_{j m_{j} 1}\right| r_{t}^{d_{j 1}} e^{\delta_{n}(A, \theta) r_{t}^{n}(1+o(1))}(1+o(1)) \\
& \leq 2\left|a_{j m_{j} d_{j m_{j}}}\right| r_{t}^{d} e^{m \delta_{n}(A, \theta) r_{t}^{n}(1+o(1))}(1+o(1)),(j \neq s)
\end{aligned}
$$

where $d=\max \left\{d_{j m_{j}}: j=0, \cdots, s-1, s+1, \cdots, k-1\right\}$. Substituting (21), (22), (24), (25) into (23), we obtain that for sufficiently large $r_{t}$

$$
\frac{1}{2}\left|a_{s m_{s} d_{s m_{s}}}\right| r_{t} d_{s m_{s}} e^{m_{s} \delta_{n}(A, \theta) r_{t}^{n}(1+o(1))}(1+o(1)) \leq C_{0} r_{t}^{d+k \rho} e^{m \delta_{n}(A, \theta)(1+o(1)) r_{t}^{n}}(1+o(1))
$$

where $C_{0}>0$ is a constant. From (26), we can get a contradiction by $m_{s}>m$ and $\delta_{n}(A, \theta)>0$, so

$$
\left|f\left(r e^{i \theta}\right)\right| \leq M r^{s} \leq M_{1} r^{k}, \quad M_{1}>0,
$$

on the ray $\arg z=\theta \in H_{n,+} \backslash E_{1}$.

Case 2. Now, we take a ray $\arg z=\theta \in H_{n,-}$, then $\delta_{n}(A, \theta)<0$. If $\left|f^{(k)}\left(r e^{i \theta}\right)\right|$ is unbounded on the ray $\arg z=\theta$, then by Lemma 3, there exists a sequence $\left\{z_{t}=r_{t} e^{i \theta}\right\}$ such that as $r_{t} \rightarrow+\infty, f^{(s)}\left(z_{t}\right) \rightarrow \infty$ and

$$
\left|\frac{f^{(i)}\left(z_{t}\right)}{f^{(k)}\left(z_{t}\right)}\right| \leq r_{t}^{k-i}(1+o(1)) \leq 2 r_{t}{ }^{k}, \quad i=0, \cdots, k-1
$$

By (7), we get

$$
-1=P_{k-1}\left(e^{A\left(z_{t}\right)}\right) \frac{f^{(k-1)}\left(z_{t}\right)}{f^{(k)}\left(z_{t}\right)}+\cdots+P_{0}\left(e^{A\left(z_{t}\right)}\right) \frac{f\left(z_{t}\right)}{f^{(k)}\left(z_{t}\right)} .
$$

For $r_{t} \rightarrow+\infty$, we have

$$
\begin{aligned}
\left|P_{j}\left(e^{A\left(z_{t}\right)}\right)\right| & =\left|a_{j m_{j}}\left(z_{t}\right) e^{m_{j} A\left(z_{t}\right)}+\cdots+a_{j 1}\left(z_{t}\right) e^{A\left(z_{t}\right)}\right| \\
& \leq\left|a_{j m_{j} d_{j m_{j}}}\right| r^{d_{j m_{j}}} e^{m_{j} \delta_{n}(A, \theta) r_{t}^{n}(1+o(1))}(1+o(1))+\cdots+\left|a_{j m_{j} 1}\right| r^{d_{j 1}} e^{\delta_{n}(A, \theta) r_{t}^{n}(1+o(1))}(1+o(1)) \\
& \leq 2\left|a_{j m_{j} 1}\right| r^{d} e^{\delta_{n}(A, \theta) r_{t}^{n}(1+o(1))}(1+o(1))(j=0, \ldots, k-1) .
\end{aligned}
$$

Substituting (28) and (30) into (29), we obtain that for sufficiently large $r_{t}$

$$
1 \leq C_{1} r_{t}^{k+d} e^{\delta_{n}(A, \theta) r_{t}^{n}(1+o(1))}(1+o(1)), \quad C_{1}>0
$$

Since $\delta_{n}(A, \theta)<0$, when $r_{t} \rightarrow+\infty$, by (31), we get $1 \leq 0$, this is a contradiction. Hence

$$
\left|f\left(r e^{i \theta}\right)\right| \leq M_{2} r^{k}, \quad M_{2}>0
$$

on the ray $\arg z=\theta \in H_{n,-} \backslash E_{1}$. From Lemma 5, (27) and (32), we know that $f(z)$ is a polynomial, which contradicts the assertion that $f(z)$ is transcendental. Therefore, $\rho(f)=\infty$.

Step 2. We prove that $\rho_{2}(f)=n$. By Lemma 7 and $\rho\left(P_{j}\left(e^{A(z)}\right)\right)=n(j=0, \cdots, k-1)$, we see that $\rho_{2}(f) \leq$ $\max \left\{\rho\left(P_{j}\left(e^{A(z)}\right)\right)\right\}=n$.

Now, we suppose that there exists a solution $f_{0}$ satisfies $\rho_{2}\left(f_{0}\right)=\alpha<n$. Then we have

$$
\limsup _{r \rightarrow+\infty} \frac{\log T\left(r, f_{0}\right)}{r^{n}}=0 .
$$


By Lemma 6, we see that there exists a subset $E_{3} \subset(1, \infty)$ having finite logarithmic measure such that for all $z$ satisfying $|z|=r \notin E_{3} \cup[0,1]$,

$$
\left|\frac{f_{0}(j)}{f_{0}(z)}\right| \leq C\left[T\left(2 r, f_{0}\right)\right]^{k+1}, \quad j=1, \cdots, k,
$$

where $C(>0)$ is some constant. From the Wiman-Valiron theory, there is a set $E_{8} \subset(1, \infty)$ having finite logarithmic measure, such that we can choose a $z$ satisfying $|z|=r \notin[0,1] \cup E_{8}$ and $\left|f_{0}(z)\right|=M\left(r, f_{0}\right)$, then we get

$$
\frac{f_{0}^{(j)}(z)}{f_{0}(z)}=\left(\frac{v(r)}{z}\right)^{j}(1+o(1)), \quad j=1, \cdots, k-1,
$$

where $v(r)$ is the central index of $f_{0}(z)$. By Lemma 9, we see that there exists a sequence $\left\{z_{t}=r_{t} e^{i \theta_{t}}\right\}$ such that $\left|f_{0}\left(z_{t}\right)\right|=M\left(r_{t}, f_{0}\right), \theta_{t} \in[0,2 \pi)$, with $r_{t} \notin[0,1] \cup E_{5} \cup E_{8}, r_{t} \rightarrow+\infty$ and for any sufficiently large $M_{3}(>2 k+3)$

$$
v\left(r_{t}\right)>r_{t}^{M_{3}}>r_{t}
$$

Case 1. Suppose $\theta_{0} \in H_{n,+}$. Since $\delta_{n}(A, \theta)=\alpha_{n} \cos \left(\theta_{n}+n \theta\right)$ is a continuous function of $\theta$, by $\theta_{t} \rightarrow \theta_{0}$ we get $\lim _{t \rightarrow \infty} \delta_{n}\left(A, \theta_{t}\right)=\delta_{n}\left(A, \theta_{0}\right)>0$. Therefore, there exists a constant $N(>0)$ such that as $t>N$,

$$
\delta_{n}\left(A, \theta_{t}\right) \geq \frac{1}{2} \delta_{n}\left(A, \theta_{0}\right)>0 .
$$

By (33), for any given $\varepsilon_{1}\left(0<\varepsilon_{1}<\frac{1}{2^{n+1}(k+1)} \delta_{n}\left(A, \theta_{0}\right)\right)$, and $t>N$,

$$
\left[T\left(2 r_{t}, f_{0}\right)\right]^{k+1} \leq e^{\varepsilon_{1}(k+1)\left(2 r_{t}\right)^{n}} \leq e^{\frac{1}{2} \delta_{n}\left(A, \theta_{t}\right) r_{t}{ }^{n}} .
$$

By (34), (35) and (37), we have

$$
\left(\frac{v\left(r_{t}\right)}{r_{t}}\right)^{k-s}(1+o(1))=\left|\frac{f_{0}^{(k-s)}\left(z_{t}\right)}{f_{0}\left(z_{t}\right)}\right| \leq C\left[T\left(2 r_{t}, f_{0}\right)\right]^{k+1} \leq C e^{\frac{1}{2} \delta_{n}\left(A, \theta_{0}\right) r_{t}{ }^{n}} .
$$

By (7), we get

$$
-\frac{f_{0}^{(s)}\left(z_{t}\right)}{f_{0}\left(z_{t}\right)} P_{s}\left(e^{A\left(z_{t}\right)}\right)=\frac{f_{0}^{(k)}\left(z_{t}\right)}{f_{0}\left(z_{t}\right)}+\sum_{j=0, j \neq s}^{k-1} P_{j}\left(e^{A\left(z_{t}\right)}\right) \frac{f_{0}^{(j)}\left(z_{t}\right)}{f_{0}\left(z_{t}\right)} .
$$

Substituting (24), (25) and (35) into (39), we get for sufficiently large $r_{t}$,

$$
\begin{aligned}
& \left(\frac{v\left(r_{t}\right)}{r_{t}}\right)^{s} \frac{1}{2}\left|a_{s m_{s} d_{s m_{s}}}\right| r_{t} d_{s m_{s}} e^{m_{s} \delta_{n}\left(A, \theta_{t}\right) r_{t}{ }^{n}(1+o(1))}(1+o(1)) \\
& \quad \leq\left(\frac{v\left(r_{t}\right)}{r_{t}}\right)^{k}(1+o(1))+\sum_{j=0, j \neq s}^{k-1} 2\left|a_{j m_{j}} d_{j m_{j}}\right| r_{t}^{d} e^{m \delta_{n}(A, \theta) r_{t}^{n}(1+o(1))}\left(\frac{v\left(r_{t}\right)}{r_{t}}\right)^{j}(1+o(1)) .
\end{aligned}
$$

By (36), (38) and (40), we get

$$
\begin{aligned}
& \left|a_{s m_{s}} d_{s m_{s}}\right| r_{t} d_{s m_{s}} e^{m_{s} \delta_{n}\left(A, \theta_{t}\right) r_{t}{ }^{n}(1+o(1))}(1+o(1)) \\
& \leq 2\left(\frac{v\left(r_{t}\right)}{r_{t}}\right)^{k-s}(1+o(1))+\sum_{j=0, j \neq s}^{k-1} 4\left|a_{j m_{j}} d_{j m_{j}}\right| r_{t}^{d} e^{m \delta_{n}(A, \theta) r_{t}^{n}(1+o(1))}\left(\frac{v\left(r_{t}\right)}{r_{t}}\right)^{j-s}(1+o(1)) \\
& \leq 2\left(\frac{v\left(r_{t}\right)}{r_{t}}\right)^{k-s}(1+o(1))+\sum_{j=0}^{s-1} 4\left|a_{j m_{j} d_{j m_{j}}}\right| r_{t}^{d} e^{m \delta_{n}(A, \theta) r_{t}^{n}(1+o(1))}\left(\frac{v\left(r_{t}\right)}{r_{t}}\right)^{j-s}(1+o(1)) \\
& +\sum_{j=s+1}^{k-1} 4\left|a_{j m_{j} d_{j m_{j}}}\right| r_{t}^{d} e^{m \delta_{n}(A, \theta) r_{t}^{n}(1+o(1))}\left(\frac{v\left(r_{t}\right)}{r_{t}}\right)^{j-s}(1+o(1))
\end{aligned}
$$




$$
\leq C_{2} r_{t}{ }^{d} e^{m \delta_{n}(A, \theta) r_{t}^{n}(1+o(1))}\left(\frac{v\left(r_{t}\right)}{r_{t}}\right)^{k-s}(1+o(1))
$$

where $C_{2}>0$ is a constant. From this inequality and (38), it follows that

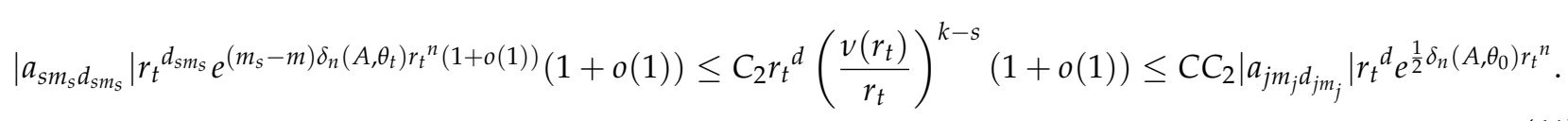

Since $m_{S}-m \geq 1>\frac{1}{2}$ and $\delta\left(A, \theta_{t}\right) \geq \frac{1}{2} \delta_{n}\left(A, \theta_{0}\right)>0$, we see that (41) is a contradiction.

Case 2. Suppose $\theta_{0} \in H_{n,-}$. Since $\delta_{n}(A, \theta)$ is a continuous function of $\theta$, by $\theta_{t} \rightarrow \theta_{0}$ we get $\lim _{t \rightarrow \infty} \delta_{n}\left(A, \theta_{t}\right)=$ $\delta_{n}\left(A, \theta_{0}\right)<0$. Therefore, there exists a constant $N(>0)$ such that as $t>N$,

$$
\delta_{n}\left(A, \theta_{t}\right) \leq \frac{1}{2} \delta_{n}\left(A, \theta_{0}\right)<0
$$

By (7), we can write

$$
e^{-m_{s} A\left(z_{t}\right)} \frac{f_{0}^{(k)}\left(z_{t}\right)}{f_{0}\left(z_{t}\right)}=e^{-m_{s} A\left(z_{t}\right)} P_{k-1}\left(e^{A\left(z_{t}\right)}\right) \frac{f^{(k-1)}\left(z_{t}\right)}{f_{0}\left(z_{t}\right)}+\cdots+e^{-m_{s} A\left(z_{t}\right)} P_{0}\left(e^{A\left(z_{t}\right)}\right) .
$$

From (6) and $\delta_{n}\left(A, \theta_{t}\right)<0$, we get

$$
\begin{aligned}
\left|e^{-m_{s} A\left(z_{t}\right)} P_{j}\left(e^{A\left(z_{t}\right)}\right)\right| & =\left|e^{-m_{s} A\left(z_{t}\right)}\left(a_{j m_{j}}\left(z_{t}\right) e^{m_{j} A\left(z_{t}\right)}+\cdots+a_{j 1}\left(z_{t}\right) e^{A\left(z_{t}\right)}\right)\right| \\
& =\left|a_{j m_{j}}\left(z_{t}\right) e^{-\left(m_{s}-m_{j}\right) A\left(z_{t}\right)}+\cdots+a_{j 1}\left(z_{t}\right) e^{-\left(m_{s}-1\right) A\left(z_{t}\right)}\right| \\
& \leq C_{3} r_{t}{ }^{d} e^{-\left(m_{s}-1\right) \delta\left(A, \theta_{t}\right) r_{t}{ }^{n}(1+o(1))}(1+o(1))
\end{aligned}
$$

where $C_{3}>0$ is a constant. Substituting (35) and (43) into (42), we get

$$
e^{-m_{s} \delta\left(A, \theta_{t}\right) r_{t}^{n}(1+o(1))} v\left(r_{t}\right) \leq C_{4} r_{t}^{d+k} e^{-\left(m_{s}-1\right) \delta\left(A, \theta_{t}\right) r_{t}^{n}(1+o(1))}(1+o(1)),
$$

where $C_{4}>0$ is a constant. By substituting (36) into (44), we have

$$
r_{t}{ }^{M_{3}} e^{-m_{s} \delta\left(A, \theta_{t}\right) r_{t}{ }^{n}(1+o(1))} \leq C_{4} r_{t}^{d+k} e^{-\left(m_{s}-1\right) \delta\left(A, \theta_{t}\right) r_{t}^{n}(1+o(1))}(1+o(1)) .
$$

Since $\delta\left(A, \theta_{t}\right) \leq \frac{1}{2} \delta_{n}\left(A, \theta_{0}\right)<0$, we see (45) is also a contradiction.

Case 3. Suppose $\theta_{0} \in H_{n, 0}$. Since $\theta_{t} \rightarrow \theta_{0}$, for any given $\varepsilon_{2}\left(0<\varepsilon_{2}<\frac{1}{10 n}\right)$, there exists as integer $N(>0)$, such that as $t>N, \theta_{t} \in\left[\theta_{0}-\varepsilon_{2}, \theta_{0}+\varepsilon_{2}\right]$, and

$$
z_{t}=r_{t} e^{i \theta_{t}} \in \bar{\Omega}=\left\{z: \theta_{0}-\varepsilon_{2} \leq \arg z \leq \theta_{0}+\varepsilon_{2}\right\}
$$

By Lemma 6, we se that there exist a subset $E_{3} \subset(1, \infty)$ having logarithmic measure $\operatorname{lm} E_{3}<\infty$, and a constant $C>0$ such that for all $z$ satisfying $|z|=r \notin E_{3} \cup[0,1]$,

$$
\left|\frac{f_{0}{ }^{(i)}(z)}{f_{0}{ }^{(s)}(z)}\right| \leq C\left[T\left(2 r, f_{0}{ }^{(s)}\right)\right]^{k-s+1}, \quad i=s+1, \cdots, k,
$$

Now, we consider the growth of $f_{0}\left(r e^{i \theta}\right)$ on a ray $\arg z=\theta \in \bar{\Omega} \backslash\left\{\theta_{0}\right\}$. By the properties of cosine function, we suppose without loss of generality that $\delta_{n}(A, \theta)>0$ for $\theta \in\left[\theta_{0}-\varepsilon_{2}, \theta_{0}\right)$ and $\delta_{n}(A, \theta)<0$ for $\theta \in\left(\theta_{0}, \theta_{0}+\varepsilon_{2}\right]$. Subcase 3.1 For a fixed $\theta \in\left[\theta_{0}-\varepsilon_{2}, \theta_{0}\right)$, we have $\delta_{n}(A, \theta)>0$. Since $\rho_{2}\left(f_{0}\right)<n$, we get that $f_{0}$ satisfies (33). From $T\left(r, f_{0}{ }^{(s)}\right)<(s+1) T\left(r, f_{0}\right)+S\left(r, f_{0}\right)$, where $S(r, f)=o(T(r, f))$, as $r \rightarrow+\infty$ outside of a possible 
exceptional set of finite logarithmic measure, we get that $f_{0}{ }^{(s)}$ also satisfies (33). So for any given $\varepsilon_{2}$ satisfying $0<\varepsilon_{2}<\frac{1}{2^{n+1}(k-s+1)} \delta_{n}(A, \theta)$, we have

$$
\left[T\left(2 r_{t}, f_{0}^{(s)}\right)\right]^{k-s+1} \leq e^{\varepsilon_{2}(k-s+1)\left(2 r_{t}\right)^{n}} \leq e^{\frac{1}{2} \delta_{n}\left(A, \theta_{0}\right) r_{t}{ }^{n}}
$$

We assert that $\left|f_{0}^{(s)}\left(r e^{i \theta}\right)\right|$ is bounded on the ray $\arg z=\theta \in\left[\theta_{0}-\varepsilon_{2}, \theta_{0}\right)$. If $\left|f^{(s)}\left(r e^{i \theta}\right)\right|$ is unbounded on the ray $\arg z=\theta$, then, by Lemma 3, there exists a sequence $\left\{y_{j}=R_{j} e^{i \theta}\right\}$ such that as $R_{j} \rightarrow \infty, f_{0}^{(s)}\left(y_{j}\right) \rightarrow \infty$ and

$$
\left|\frac{f_{0}^{(i)}\left(y_{j}\right)}{f_{0}^{(s)}\left(y_{j}\right)}\right| \leq R_{j}^{s-i}(1+o(1)) \leq 2 R_{j}^{s}, \quad i=0, \cdots, s-1 .
$$

By Remark 5 and $f_{0}^{(s)}\left(y_{j}\right) \rightarrow \infty$, we know that $\left|y_{j}\right|=R_{j} \notin E_{4}$. By (46) and (47), we have for sufficiently large $j$,

$$
\left|\frac{f_{0}^{(j)}\left(y_{j}\right)}{f_{0}^{(s)}\left(y_{j}\right)}\right| \leq C\left[T\left(2 R_{j}, f_{0}^{(s)}\right)\right]^{k-s+1} \leq C e^{\frac{1}{2} \delta_{n}\left(A, \theta_{0}\right) R_{j}^{n}}, \quad j=s+1, \cdots, k .
$$

Substituting (24), (25), (48) and (49) into (23)

$$
\begin{aligned}
& \frac{1}{2}\left|a_{s m_{s} d_{s m_{s}}}\right| R_{j} d_{s m_{s}} e^{m_{s} \delta_{n}(A, \theta) R_{j}^{n}(1+o(1))}(1+o(1)) \\
& =\left|P_{S}\left(e^{A\left(y_{j}\right)}\right)\right| \\
& \leq\left|\frac{f^{(k)}\left(y_{j}\right)}{f^{(s)}\left(y_{j}\right)}\right|+\sum_{j=0 j \neq s}^{k-1}\left|P_{j}\left(e^{A\left(y_{j}\right)}\right)\right| \frac{f^{(j)}\left(y_{j}\right)}{f^{(s)}\left(y_{j}\right)} \mid \\
& =\left|\frac{f^{(k)}\left(y_{j}\right)}{f^{(s)}\left(y_{j}\right)}\right|+\sum_{j=0}^{s-1}\left|P_{j}\left(e^{A\left(y_{j}\right)}\right)\right| \frac{f^{(j)}\left(y_{j}\right)}{f^{(s)}\left(y_{j}\right)}\left|+\sum_{j=s+1}^{k-1}\right| P_{j}\left(e^{A\left(y_{j}\right)}\right)|| \frac{f^{(j)}\left(y_{j}\right)}{f^{(s)}\left(y_{j}\right)} \mid \\
& \leq C e^{\frac{1}{2} \delta_{n}\left(A, \theta_{0}\right) R_{j}^{n}}+\sum_{j=0}^{s-1} 4\left|a_{j m_{j} d_{j m_{j}}}\right| R_{j}^{d} e^{m \delta_{n}(A, \theta) R_{j}^{n}(1+o(1))} R_{j}^{s}(1+o(1)) \\
& +\sum_{j=s+1}^{k-1} 2\left|a_{j m_{j} d_{j m_{j}}}\right| R_{j}^{d} e^{m \delta_{n}(A, \theta) R_{j}^{n}(1+o(1))} C e^{\frac{1}{2} \delta_{n}\left(A, \theta_{0}\right) R_{j}^{n}} \\
& \leq C_{5} R_{j}^{d} e^{\left(\frac{1}{2}+m\right) \delta_{n}(A, \theta) R_{j}{ }^{n}},
\end{aligned}
$$

where $C_{5}>0$ is a constant, which yields a contradiction by $m_{s}-m \geq 1>\frac{1}{2}$ and $\delta_{n}(A, \theta)>0$. Hence $\left|f_{0}^{(s)}\left(r e^{i \theta}\right)\right|$ is bounded on the ray $\arg z=\theta$, so

$$
\left|f_{0}\left(r e^{i \theta}\right)\right| \leq M_{4} r^{s}, \quad M_{4}>0,
$$

on the ray $\arg z=\theta \in\left[\theta_{0}-\varepsilon_{4}, \theta_{0}\right)$.

Subcase 3.2 For a fixed $\theta \in\left(\theta_{0}, \theta_{0}+\varepsilon_{2}\right]$, we have $\delta_{n}(A, \theta)<0$. Using a reasoning similar to that in Subcase 3.1, we obtain

$$
\left|f_{0}\left(r e^{i \theta}\right)\right| \leq M_{5} r^{k}, \quad M_{5}>0,
$$

on the ray $\arg z=\theta \in\left(\theta_{0}, \theta_{0}+\varepsilon_{4}\right]$. By (51) and (52), we see that on the ray $\arg z=\theta \in \bar{\Omega} \backslash\left\{\theta_{0}\right\}$,

$$
\left|f_{0}\left(r e^{i \theta}\right)\right| \leq M_{5} r^{k}, \quad M_{5}>0 .
$$

But since $\rho\left(f_{0}\left(r e^{i \theta}\right)\right)=\infty$ and $\left\{z_{t}=r_{t} e^{i \theta_{t}}\right\}$ satisfies $\left|f_{0}\left(z_{t}\right)\right|=M\left(r_{t}, f_{0}\right)$, we see that, for any large $M_{6}(>k)$, as $t$ is sufficiently large,

$$
\left|f_{0}\left(z_{t}\right)\right|=\left|f_{0}\left(z_{t}\right)\right|=\left|f_{0}\left(z_{t}\right)\right|=\left|f_{0}\left(r_{t} e^{i \theta_{t}}\right)\right| \geq \exp \left\{r_{t}^{M_{6}}\right\}
$$


Since $z_{t} \in \bar{\Omega}$, by (53) and (54), we see that $\theta_{t}=\theta_{0}$ as $t \rightarrow \infty$. Therefore, $\delta_{n}\left(A, \theta_{t}\right)=0$ as $t \rightarrow \infty$. Thus, for sufficiently large $t$,

$$
\begin{aligned}
\left|P_{j}\left(e^{z_{t}}\right)\right| & =\left|a_{j m_{j}}\left(z_{t}\right) e^{m_{j} A\left(z_{t}\right)}+a_{j m_{j-1}}\left(z_{t}\right) e^{m_{j-1} A\left(z_{t}\right)}+\cdots+a_{j 1}\left(z_{t}\right) e^{A\left(z_{t}\right)}\right| \\
& \leq\left|a_{j m_{j}}\left(z_{t}\right)\right|+\left|a_{j m_{j-1}}\left(z_{t}\right)\right|+\cdots+\left|a_{j 1}\left(z_{t}\right)\right| \leq C_{6} r^{d},
\end{aligned}
$$

where $j=0, \cdots, k-1$ and $C_{6}>0$ is a constant. By (7), (35) and (55), we get that

$$
\left|-\left(\frac{v\left(r_{t}\right)}{z_{t}}\right)^{k}(1+o(1))\right|=\left|-\frac{f_{0}^{(k)}\left(z_{t}\right)}{f_{0}\left(z_{t}\right)}\right| \leq C_{7} r^{d}\left(\frac{v\left(r_{t}\right)}{z_{t}}\right)^{k-1}(1+o(1)),
$$

i.e.,

$$
v\left(r_{t}\right)(1+o(1)) \leq C_{7} r^{d+1}(1+o(1)),
$$

where $C_{7}>0$ is a constant. Substituting (36) into (56), we obtain also a contradiction. So we have $\rho_{2}(f)=$ $n$.

Proof of Corollary 1. From Theorem 7, we get $\rho(f)=\infty$ and $\rho_{2}(f)=n$. Let $g=f-z$, then $f=g+z$. Substituting it into (7), we have

$$
g^{(k)}+P_{k-1}\left(e^{A(z)}\right) g^{(k-1)}+\cdots+P_{0}\left(e^{A(z)}\right) g=-z P_{0}\left(e^{A(z)}\right)-P_{1}\left(e^{A(z)}\right) .
$$

Since $-z P_{0}\left(e^{A(z)}\right)-P_{1}\left(e^{A(z)}\right) \not \equiv 0$, from Lemma 11, $\rho(g)=\infty$ and $\rho_{2}(g)=n$ we conclude $\bar{\lambda}(g)=\lambda(g)=$ $\rho(g)=\infty$ and $\bar{\lambda}_{2}(g)=\lambda_{2}(g)=\rho_{2}(g)=n$. So $\bar{\tau}(f)=\tau(f)=\rho(f)=\infty$ and $\bar{\tau}_{2}(f)=\tau_{2}(f)=\rho_{2}(f)=n$.

Proof of Theorem 8. From Theorem 7, we get $\rho(f)=\infty$ and $\rho_{2}(f)=n$.

(i) Let $g=f-z$, then $f=g+z$. Substituting it into (8), we have

$$
g^{\prime \prime}+P\left(e^{A(z)}\right) g^{\prime}+Q\left(e^{A(z)}\right) g=-P\left(e^{A(z)}\right)-z Q\left(e^{A(z)}\right) .
$$

Since $p \neq s$, we get $-P\left(e^{A(z)}\right)-Q\left(e^{A(z)}\right) z \not \equiv 0$. From Lemma 11, we obtain $\lambda(g)=\rho(g)=\rho(f)=\infty$ and $\lambda_{2}(g)=\rho_{2}(g)=\rho_{2}(f)=n$. So $\lambda(f-z)=\infty$ and $\lambda_{2}(f-z)=n$.

(ii) Differentiating both sides of (8), we get that

$$
f^{\prime \prime \prime}+P\left(e^{A(z)}\right) f^{\prime \prime}+\left[\left(P\left(e^{A(z)}\right)\right)^{\prime}+Q\left(e^{A(z)}\right)\right] f^{\prime}+\left(Q\left(e^{A(z)}\right)\right)^{\prime} f=0 .
$$

By (8), we have

$$
f=-\frac{f^{\prime \prime}+P\left(e^{A(z)}\right) f^{\prime}}{Q\left(e^{A(z)}\right)}
$$

Substituting (58) into (57), we get

$$
f^{\prime \prime \prime}+\left[\left(P\left(e^{A(z)}\right)\right)^{\prime}-\frac{\left(Q\left(e^{A(z)}\right)\right)^{\prime}}{Q\left(e^{A(z)}\right)}\right] f^{\prime \prime}+\left[\left(P\left(e^{A(z)}\right)\right)^{\prime}+Q\left(e^{A(z)}\right)-\frac{\left(Q\left(e^{A(z)}\right)\right)^{\prime}}{Q\left(e^{A(z)}\right)} P\left(e^{A(z)}\right)\right] f^{\prime}=0 .
$$

Let $g=f^{\prime}-z$, then $f^{\prime}=g+z, f^{\prime \prime}=g^{\prime}+1, f^{\prime \prime \prime}=g^{\prime \prime}$. Substituting these into (59), we get that

$$
\begin{aligned}
g^{\prime \prime} & +\left[\left(P\left(e^{A(z)}\right)\right)^{\prime}-\frac{\left(Q\left(e^{A(z)}\right)\right)^{\prime}}{Q\left(e^{A(z)}\right)}\right] g^{\prime}+\left[\left(P\left(e^{A(z)}\right)\right)^{\prime}+Q\left(e^{A(z)}\right)-\frac{\left(Q\left(e^{A(z)}\right)\right)^{\prime}}{Q\left(e^{A(z)}\right)} P\left(e^{A(z)}\right)\right] g \\
& =-P\left(e^{A(z)}\right)+\frac{\left(Q\left(e^{A(z)}\right)\right)^{\prime}}{Q\left(e^{A(z)}\right)}-\left[\left(P\left(e^{A(z)}\right)\right)^{\prime}+Q\left(e^{A(z)}\right)-\frac{\left(Q\left(e^{A(z)}\right)\right)^{\prime}}{Q\left(e^{A(z)}\right)} P\left(e^{A(z)}\right)\right] z \\
& =h(z) .
\end{aligned}
$$


Next, we prove that $h(z) \not \equiv 0$. If $h(z) \equiv 0$, then

$$
-P\left(e^{A(z)}\right)+\frac{\left(Q\left(e^{A(z)}\right)\right)^{\prime}}{Q\left(e^{A(z)}\right)} \equiv\left[\left(P\left(e^{A(z)}\right)\right)^{\prime}+Q\left(e^{A(z)}\right)-\frac{\left(Q\left(e^{A(z)}\right)\right)^{\prime}}{Q\left(e^{A(z)}\right)} P\left(e^{A(z)}\right)\right] z .
$$

Since $Q(z) \not \equiv 0$, we have

$$
\left(Q\left(e^{A(z)}\right)\right)^{\prime}-\left(Q\left(e^{A(z)}\right)\right)^{2} z \equiv P\left(e^{A(z)}\right) Q\left(e^{A(z)}\right)+\left[\left(P\left(e^{A(z)}\right)\right)^{\prime} Q\left(e^{A(z)}\right)-\left(Q\left(e^{A(z)}\right)\right)^{\prime} P\left(e^{A(z)}\right)\right] z .
$$

Suppose $p>s$. By taking $z=r$, we have

$$
P\left(e^{A(r)}\right)=a_{p}(r) e^{p A(r)}+\cdots+a_{1}(r) e^{A(r)}, \quad \text { and } \quad Q\left(e^{A(r)}\right)=b_{s}(r) e^{s A(r)}+\cdots+b_{1}(r) e^{A(r)} .
$$

We get

$$
\begin{aligned}
\left(P\left(e^{A(r)}\right)\right)^{\prime} & =\sum_{j=1}^{p}\left(a_{j}^{\prime}(r)+j A^{\prime}(r) a_{j}(r)\right) e^{j A(r)} \\
& =\left(a_{p}^{\prime}(r)+p A^{\prime}(r) a_{p}(r)\right) e^{p A(r)}+\cdots+\left(a_{1}^{\prime}(r)+A^{\prime}(r) a_{1}(r)\right) e^{A(r)}
\end{aligned}
$$

and

$$
\begin{aligned}
\left(Q\left(e^{A(r)}\right)\right)^{\prime} & =\sum_{j=1}^{s}\left(b_{j}^{\prime}(r)+j A^{\prime}(r) b_{j}(r)\right) e^{j A(r)} \\
& =\left(b_{s}^{\prime}(r)+s A^{\prime}(r) b_{s}(r)\right) e^{s A(r)}+\cdots+\left(b_{1}^{\prime}(r)+A^{\prime}(r) b_{1}(r)\right) e^{A(r)} .
\end{aligned}
$$

So, we obtain

$$
\begin{aligned}
& \left|P\left(e^{A(r)}\right) Q\left(e^{A(r)}\right)+\left(P\left(e^{A(r)}\right)\right)^{\prime} Q\left(e^{A(r)}\right) r-\left(Q\left(e^{A(r)}\right)\right)^{\prime} P\left(e^{A(r)}\right) r\right| \\
& =\left|a_{p}(r) b_{s}(r)+(p-s) r A^{\prime}(r) a_{p}(r) b_{s}(r)+\left(a_{p}^{\prime}(r) b_{s}(r)-a_{p}(r) b_{s}^{\prime}(r)\right) r\right| e^{(p+s) R e c_{n} r^{n}(1+o(1))}(1+o(1)) .
\end{aligned}
$$

Since $a_{p}(r), b_{s}(r)$ and $A(r)$ are polynomials and $p>s$, we get

$$
\operatorname{deg}\left((p-s) r A^{\prime}(r) a_{p}(r) b_{s}(r)\right)>\operatorname{deg}\left[a_{p}(r) b_{s}(r)+\left(a_{p}^{\prime}(r) b_{s}(r)-a_{p}(r) b_{s}^{\prime}(r)\right) r\right] .
$$

So, we have

$$
\left|(p-s) r A^{\prime}(r) a_{p}(r) b_{s}(r)+a_{p}(r) b_{s}(r)+\left(a_{p}^{\prime}(r) b_{s}(r)-a_{p}(r) b_{s}^{\prime}(r)\right) r\right|=M r^{d_{1}}(1+o(1)) \not \equiv 0,
$$

where $M>0$ and $d_{1}>0$ are some constants. It follows that

$$
\left|P\left(e^{A(r)}\right) Q\left(e^{A(r)}\right)+\left(P\left(e^{A(r)}\right)\right)^{\prime} Q\left(e^{A(r)}\right) r-\left(Q\left(e^{A(r)}\right)\right)^{\prime} P\left(e^{A(r)}\right) r\right|=M r^{d_{1}} e^{(p+s) \operatorname{Rec}_{n} r^{n}(1+o(1))}(1+o(1)) .
$$

From (61), we have

$$
\begin{aligned}
M r^{d_{1}} e^{(p+s) \operatorname{Rec}_{n} r^{n}(1+o(1))}(1+o(1)) & =\left|P\left(e^{A(r)}\right) Q\left(e^{A(r)}\right)+\left(P\left(e^{A(r)}\right)\right)^{\prime} Q\left(e^{A(r)}\right) r-\left(Q\left(e^{A(r)}\right)\right)^{\prime} P\left(e^{A(r)}\right) r\right| \\
& =\left|\left(Q\left(e^{A(r)}\right)\right)^{\prime}-\left(Q\left(e^{A(r)}\right)\right)^{2} r\right| \leq M_{1} r^{d_{2}} e^{2 s \operatorname{Rec}_{n} r^{n}(1+o(1))}(1+o(1)),
\end{aligned}
$$

where $M_{1}>0$ and $d_{2}>0$ are some constants, which is a contradiction. So we have $h(z) \not \equiv 0$. If $p<s$, by (61) for $z=r$ we have

$$
\begin{aligned}
M_{2} r^{d_{3}} e^{2 s \operatorname{Rec}_{n} r^{n}(1+o(1))}(1+o(1)) & =\left[\left(Q\left(e^{A(r)}\right)\right)^{2}+\left(P\left(e^{A(r)}\right)\right)^{\prime} Q\left(e^{A(r)}\right)-\left(Q\left(e^{A(r)}\right)\right)^{\prime} P\left(e^{A(r)}\right)\right] r \\
& =\left|\left(Q\left(e^{A(r)}\right)\right)^{\prime}-P\left(e^{A(r)}\right) Q\left(e^{A(r)}\right)\right| \\
& \leq M_{3} r^{d_{4}} e^{(p+s) \operatorname{Rec}_{n} r^{n}(1+o(1))}(1+o(1)),
\end{aligned}
$$


where $M_{2}>0, d_{3}>0, M_{3}>0$ and $d_{4}>0$ are some constants.This is a contradiction. So, we obtain $h(z) \not \equiv 0$. Hence, if $p \neq s$ we have $h(z) \not \equiv 0$. From Lemma 11, we get $\lambda(g)=\rho(g)=\rho\left(f^{\prime}-z\right)=\rho(f)=\infty$ and $\lambda_{2}(g)=\rho_{2}(g)=\rho_{2}\left(f^{\prime}-z\right)=\rho_{2}(f)=n$.

Proof of Theorem 9. Suppose that $f \not \equiv 0$ is a solution of (10). Since $\rho\left(P^{*}\right)=\rho\left(Q^{*}\right)=n$, then by Lemma 7, we see that

$$
\rho_{2}(f) \leq \max \left\{\rho\left(P^{*}\right), \rho\left(Q^{*}\right)\right\}=n .
$$

By Lemma 6, we se that there exist a subset $E_{3} \subset(1, \infty)$ having logarithmic measure $\operatorname{lm} E_{3}<\infty$, and a constant $C>0$ such that for all $z$ satisfying $|z|=r \notin E_{3} \cup[0,1]$,

$$
\left|\frac{f^{(j)}(z)}{f(z)}\right| \leq C[T(2 r, f)]^{j+1}, \quad j=1,2 .
$$

Taking $z=r$, in (2) and (3), we obtain that for sufficiently large $r$

$$
\begin{aligned}
\left|P^{*}\left(e^{A(r)}\right)\right| & =\left|a_{p}(r) e^{p A(r)}+\cdots+a_{1}(r) e^{A(r)}+a_{0}(r)\right| \\
& \leq 2\left|a_{p d_{p}}\right| r^{d_{p}} e^{p \operatorname{Rec}_{n} r^{n}(1+o(1))}(1+o(1)),
\end{aligned}
$$

and

$$
\begin{aligned}
\left|Q^{*}\left(e^{A(r)}\right)\right| & =\left|b_{s}(r) e^{s A(r)}+\cdots+b_{1}(r) e^{A(r)}+b_{0}(r)\right| \\
& \geq \frac{1}{2}\left|b_{s m_{s}}\right| r^{m_{s}} e^{s \operatorname{Rec}_{n} r^{n}(1+o(1))}(1+o(1)) .
\end{aligned}
$$

Substituting (63)-(65) into (10), we deduce that for all $z$ satisfying $|z|=r \notin E_{3} \cup[0,1]$

$$
\begin{aligned}
\frac{1}{2}\left|b_{s m_{s}}\right| r^{m_{s}} e^{S \operatorname{Rec} r_{n} r^{n}(1+o(1))}(1+o(1)) & \leq\left|\frac{f^{\prime \prime}(z)}{f(z)}+P^{*}\left(e^{A(z)}\right) \frac{f^{\prime}(z)}{f(z)}\right| \\
& \leq\left|\frac{f^{\prime \prime}(z)}{f(z)}\right|+\left|P^{*}\left(e^{A(z)}\right)\right|\left|\frac{f^{\prime}(z)}{f(z)}\right| \\
& \leq C[T(2 r, f)]^{3}+2\left|a_{p d_{p}}\right| r^{d_{p}} e^{p \operatorname{Rec} r r^{n}(1+o(1))} C[T(2 r, f)]^{2}(1+o(1)) \\
& \leq 3 C\left|a_{p d_{p}}\right| r^{d_{p}} e^{p \operatorname{Rec}_{n} r^{n}(1+o(1))}[T(2 r, f)]^{3}(1+o(1)) .
\end{aligned}
$$

By (66), we deduce that for all $z$ satisfying $|z|=r \notin E_{3} \cup[0,1]$

$$
\left|b_{s m_{s}}\right| r^{m_{s}-d_{p}} e^{(s-p) \operatorname{Rec}_{n} r^{n}(1+o(1))}(1+o(1)) \leq 6 C\left|a_{p d_{p}}\right|[T(2 r, f)]^{3}(1+o(1)) .
$$

Since $s-p>0$, by (67) and Lemma 12, we get

$$
\rho(f) \geq \limsup _{r \rightarrow+\infty} \frac{\log T(r, f)}{\log r}=+\infty, \rho_{2}(f) \geq \limsup _{r \rightarrow+\infty} \frac{\log \log T(r, f)}{\log r}=n .
$$

From (62) and (68) we obtain $\rho(f)=+\infty$ and $\rho_{2}(f)=n$.

Acknowledgments: This paper was supported by Directorate-General for Scientific Research and Technological Development(DGRSDT).

Author Contributions: All authors contributed equally to the writing of this paper. All authors read and approved the final manuscript.

Conflicts of Interest: "The authors declare no conflict of interest."

\section{References}

[1] Hayman, W. K. (1964). Meromorphic Functions (Vol. 78). Oxford Mathematical Monographs Clarendon Press, Oxford. 
[2] Laine, I. (1993). Nevanlinna Theory and Complex Differential Equations. de Gruyter Studies in Mathematics, 15. Walter de Gruyter \& Co., Berlin-New York.

[3] Yang, C. C., \& Yi, H. X. (2003). Uniqueness Theory of Meromorphic Functions (Vol. 557), Mathematics and its Applications, 557. Kluwer Academic Publishers Group, Dordrecht.

[4] Li, N., Qi, X., \& Yang, L. (2019). Some results on the solutions of higher-order linear differential equations. Bulletin of the Malaysian Mathematical Sciences Society, 42(5), 2771-2794.

[5] Wittich, H. (1967). Subnormale Lösungen der Differentialgleichung: $w^{\prime \prime}+p\left(e^{z}\right) w^{\prime}+q\left(e^{z}\right) w=0$. Nagoya Mathematical Journal, 30, 29-37.

[6] Gundersen, G. G., \& Steinbart, E. M. (1994). Subnormal solutions of second order linear differential equations with periodic coefficients. Results in Mathematics, 25(3), 270-289.

[7] Chen, Z. X., \& Shon, K. H. (2010). The hyper order of solutions of second order differential equations and subnormal solutions of periodic equations. Taiwanese Journal of Mathematics, 14(2), 611-628.

[8] Belaïdi, B., \& Zemirni, M. A. (2015). Nonexistence of subnormal solutions for a class of higher order complex differential equations. Bulletin of the Transilvania University of Braşov. Mathematics, Informatics, Physics. Series III, $8(2), 29-50$.

[9] Chen, Z. X., \& Shon, K. H. (2010, January). On subnormal solutions of periodic differential equations. In Abstract and Applied Analysis (Vol. 2010), Article ID 170762.

[10] Chen, Z. X., \& Shon, K. H. (2011). Numbers of subnormal solutions for higher order periodic differential equations. Acta Mathematica Sinica, English Series, 27(9), 1753-1768.

[11] Yang, L., \& Li, N. (2013). The hyper order and fixed points of solutions of linear differential equations. Electronic Journal of Qualitative Theory of Differential Equations, 2013(19), 1-17.

[12] Li, N., \& Yang, L. (2014). Growth of solutions to second-order complex differential equations. Electronic Journal of Differential Equations, 2014(51), 1-12.

[13] Liu, K., \& Yang, L. Z. (2009). On the complex oscillation of higher order linear differential equations. Bulletin of the Korean Mathematical Society, 46(4), 607-615.

[14] Gundersen, G. G. (1988). Finite order solutions of second order linear differential equations. Transactions of the American Mathematical Society, 305(1), 415-429.

[15] Wang, J., \& Laine, I. (2009). Growth of solutions of nonhomogeneous linear differential equations. Abstract and Applied Analysis (Vol. 2009), Article. ID 363927.

[16] Gundersen, G. G. (1988). Estimates for the logarithmic derivative of a meromorphic function, plus similar estimates. Journal of the London Mathematical Society, 2(1), 88-104.

[17] Chen, Z. (2003). On the hyper order of solutions of higher order differential equations. Chinese Annals of Mathematics, 24(4),501-508.

[18] Chen, Z. X. (2002). The growth of solutions of $f^{\prime \prime}+e^{-z} f^{\prime}+Q(z) f=0$ where the order $(Q)=1$. Science in China Series A: Mathematics, 45(3), 290-300.

[19] Chen, Z. X., \& Yang, C. C. (1999). Some further results on the zeros and growths of entire solutions of second order linear differential equations. Kodai Mathematical Journal, 22(2), 273-285.

[20] Langley, J. K. (2006). Integer points of entire functions. Bulletin of the London Mathematical Society, 38(2), $239-249$.

[21] Belaïdi, B. (2008). Growth and oscillation theory of solutions of some linear differential equations. Matematicki Vesnik, $60(4), 233-246$.

[22] Zong-Xuan, C. (1994). Zeros of meromorphic solutions of higher order linear differential equations. Analysis, 14(4), 425-438.

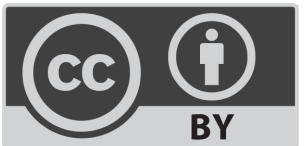

(C) 2021 by the authors; licensee PSRP, Lahore, Pakistan. This article is an open access article distributed under the terms and conditions of the Creative Commons Attribution (CC-BY) license (http://creativecommons.org/licenses/by/4.0/). 